\title{
Evaluation of the effectiveness of a Virtual Reality-based exercise program for Unilateral Peripheral Vestibular Deficit
}

\author{
Oskar Rosiak $^{\mathrm{a}, *}$, Krzysztof Krajewski $^{\mathrm{a}}$, Marek Woszczak ${ }^{\mathrm{b}}$ and Magdalena Jozefowicz-Korczynska ${ }^{\mathrm{a}}$ \\ ${ }^{a}$ Department of Otolaryngology, Balance Disorders Unit, Medical University of Lodz, \\ The Norbert Barlicki Memorial Teaching Hospital, Lodz, Poland \\ ${ }^{\mathrm{b}}$ Department of Rehabilitation, The Norbert Barlicki Memorial Teaching Hospital, Lodz, Poland
}

Received 12 February 2018

Accepted 4 December 2018

\begin{abstract}
.
BACKGROUND: Recently, two types of movement sensors have been introduced into Virtual Reality (VR) therapy: motion trackers and force-plate platforms. Combining these two methods could produce better rehabilitation outcomes. Such devices, encompassing motion trackers and force platforms, are referred to as "hybrid" VR units.

OBJECTIVE: To assess the effectiveness of a low-cost hybrid VR based vestibular rehabilitation program

METHODS: A prospective, non-randomized, controlled group study comparing training using a hybrid VR unit (Group $1 n=25$ ) vs. static posturography with visual feedback (Group $2 n=25$ ) in patients with peripheral vestibular dysfunction was conducted. The subjects underwent 10 training sessions over 10 days (30 minute sessions). All were examined on a posturography platform at the start and 1 month after rehabilitation and completed the Vertigo Symptom Scale - Short Form (VSS-SF) questionnaire.

RESULTS: Both groups demonstrated improvement in posturographic parameters, which were statistically significant, but when comparing results between both groups there were no differences. The patients reported improvement in their subjective perception of symptoms on the VSS-SF scale, which were statistically significant in both groups, but greater in the VR group. CONCLUSIONS: Both methods reduce postural sway, however subjective reduction of symptoms was greater in the VR group.
\end{abstract}

Keywords: Vertigo, vestibular rehabilitation, Virtual Reality

\section{Introduction}

Peripheral vestibular loss is the most common cause of severe vertigo and nausea. These symptoms are often accompanied by the sudden onset of spontaneous nystagmus beating towards the healthy side and disequilibrium. Patients experience fear and anxiety during or in between the attacks, which results in

\footnotetext{
${ }^{*}$ Corresponding author: Oskar Rosiak, Department of Otolaryngology, Balance Disorders Unit, Medical University of Lodz, The Norbert Barlicki Memorial Teaching Hospital, Lodz, Poland. Tel.: +48 501063456; E-mail: orosiak@me.com.
}

absence from work and a cost to society [19]. Pharmacological treatment is recommended only in the acute stage for a short time, and a further decrease of vertigo is achieved with targeted rehabilitation therapy.

Vestibular Rehabilitation Therapy (VRT) began with Cawthorne-Cooksey exercises given to patients with labyrinth deficiency after head trauma [2,3]. The main principle of VRT is to promote a natural vestibular recovery process, generally attributed to the vestibular compensation phenomenon, which is achieved by adaptation, habituation and substitution mechanisms, as defined in the works of Herdman 
[9] and Deveze et al. [4]. Correction of unbalanced vestibular, proprioceptive and visual inputs is obtained by auditory and visual feedback or optokinetic training combined with physical exercise involving upper body, head and eye movement. VRT is a non-invasive, safe and effective treatment in patients with acute or chronic peripheral vestibular loss. Patients with peripheral lesions but poor spontaneous vestibular compensation respond well to this form of therapy [7]. Conventional VRT exercises must be performed several times, amounting to $20-40$ minutes of exercise per day [8], which some patients find repetitive and monotonous. Furthermore, it is difficult for patients to receive feedback while training at home. Recent advances in technology have made it possible to exercise at home with the use of virtual reality.

Virtual Reality (VR) is a widely applied technique for generating a virtual environment using various forms of display - spherical, flat screen or head-mounted. The user can interact with objects in VR using his body movement [1]. VR has recently gained in popularity in medicine with the rapid development of mobile and visual technologies. It has been successfully applied in various medical specialties, for example: psychiatry, in treating anxiety [5], schizophrenia [18] and cognitive impairments; post-stroke hemiplegia [13]; or in pediatrics, in the rehabilitation of cerebral palsy [14].

Recently, VR was introduced to vestibular rehabilitation with various devices and protocols in which VR techniques had a similar effect to conventional vestibular therapy $[1,6]$. Some of the implemented techniques utilize expensive equipment, which limits and delays treatment. Only a few studies have explored the application of commercially available, low-cost VR systems in VRT $[15,16]$. These studies have focused either on force-plate technologies (Nintendo Balance Board) or motion sensor dependent devices, such as the Microsoft XBOX 360 Kinect, which our Department has studied in vestibular rehabilitation [11]. The motivational and enjoyment aspect of virtual reality-based programs may result in better compliance with exercises than conventional therapy [9]. With low costs, this method remains more affordable for rehabilitation centers than certified static posturography, and it is possible to introduce it as home therapy for maintaining vestibular compensation.

The hybrid VR devices defined by Herdman et al. [9] are built from accessories derived from commercially available technologies and combine motion sensors, force plate technology and a display for providing visual feedback and generating the VR environment. This system detects movement from the upper part of the body and measures the center of pressure displacement. By evoking a wider range of movements from the patient hybrid VR systems might have a better effect on postural control and vertigo. There is no research in the available literature regarding hybrid VR technology in vestibular rehabilitation; therefore, the aim of this study was to evaluate the effectiveness of a low-cost hybrid VR based vestibular rehabilitation program in a unilateral peripheral vestibular impairment.

\section{Material and methods}

A prospective, non-randomized, controlled group study comparing training using a hybrid VR vs. static posturography with visual feedback in patients with peripheral vestibular dysfunction was conducted. The study was approved of the Local Ethics Committee at the Medical University of Lodz (no. RNN/88/17/KE). All subjects provided written informed consent. All clinical investigation was conducted according to the principles expressed in the Declaration of Helsinki.

Patients who were diagnosed with vertigo and balance instability at the Balance Disorders Unit, Otolaryngology Department, Medical University of Lodz and fulfilled the study requirements were subject to a prospective analysis. The inclusion criteria were: (1) persistent vertigo and disequilibrium with unsatisfactory spontaneous compensation at least two months post-onset; (2) unilateral peripheral vestibular impairment confirmed by videonystagmography (VNG) (Ulmer SYNAPSIS 2008) as canal paresis with directional preponderance. The VNG examination assessed spontaneous ocular movements with eyes open and closed, positional tests, ocular-motor tests - smooth pursuit, optokinetic and saccadic tests, kinetic stimulation with torsion swing test and caloric test by the Fitzgerald-Hallpike method. Patients after orthopedic surgery, with a history of epilepsy, bilateral peripheral vestibular loss or central vestibular disorder were excluded.

Fifty patients were assigned to the study groups using an alternating sequence, wherein every second individual enrolled (e.g., 1, 3, 5, etc.) was assigned to the intervention group (the Virtual Reality-based exercise program - Group 1) and the alternate patients (e.g., 2, 4, 6, etc.) were assigned to the control group 
(Static posturography with visual feedback training - Group 2)

Group 1 included 25 patients, 14 women and 11 men, aged 26 to 64 years (average age $46.48 \pm 10.6$ ). Patients received ten training sessions lasting 30 minutes for two weeks using a hybrid VR unit consisting of a force plate, an upper body movement motion sensor and a central unit with a flat screen display (Neuroforma 2016, manufactured by Titanis Sp. z o.o. Warsaw, Poland).

Standing 1.5-2 meters from the display on the force plate, patients performed a set of exercises coordinating upper body movement and maintaining the center of pressure (COP) in a predetermined range or shifting the COP towards indicated positions. The software utilized includes 8 exercises for balance with 28 difficulty levels, gradually progressing from a predetermined setting according to the patient's performance. Furthermore, the range of motion on the force plate could be altered individually, depending on the affected side, thus, requiring the patient to lean more to the right in right-sided vestibular dysfunctions.

To ensure correct performance, the training was supervised by a physiotherapist. For protection purposes, the ground surface was soft, and a railing of 1.5 by 1.5 meters was put in place around the patient.

The exercise protocol consisted of two tasks. The first task was "Meteorites", a VR game set in space where a marker on the display projected the patient's COP. By moving the marker, the patient could target randomly generated, moving meteorites and satellites. The goal of the exercise was to eliminate the meteorites, thus gaining points and progressing to the next level. If a satellite was hit, the patient lost a point. Successive difficulty levels increased the speed of the objects and, simultaneously, the number of meteorites on screen.

The goal of the second task was to place hats on hangers with matching colors. The movement and selection of the hat were controlled by elevating the upper extremities and dragging the selected item across the screen to the desired location. During the exercise, the patient's COP was projected on the display in a circle, and upon reaching the periphery, the color of the circle changed from green to red. If the COP exceeded the boundaries, the hat returned to its original position. Advancing further narrowed the COP limit and extended the upper extremity range by increasing the distance between the hats and the hangers.
Group 2 included 25 people, 13 women and 12 men, aged 29 to 68 years (average age $45.20 \pm 11.07$ ). Patients received a total of ten sessions of static posturography training with visual feedback over a two-week period under the supervision of a physiotherapist. The patient stood on a firm surface with heels $5 \mathrm{~cm}$ apart and toes apart at a 45-degree angle. A display providing visual feedback was in front of the patient. The screen projected the patient's COP on a statokinesiogram. The task was to steer the COP representation towards a randomly generated point on the screen. Points were generated at 20-second intervals, and if the patient did not manage to reach the point, a new point was generated elsewhere. This cycle was repeated 86 times, and a total score was calculated at the end of exercise. The duration of each session was, on average, 25 minutes.

Throughout the course of the rehabilitation, both groups were instructed by physiotherapists on how to perform Cawthorne-Cooksey exercises at home and were asked to exercise three times daily.

Each group was examined before rehabilitation and one month after rehabilitation. The examination involved posturographic assessment on a static platform (Euroclinic SSS ED 8000) including a quiet stance with eyes open and eyes closed for $30 \mathrm{sec}-$ onds. The feet were positioned with heels $5 \mathrm{~cm}$ apart and toes at a 45-degree angle. Each test was repeated three times, and the average score was recorded. The total length and surface of the COP in both tests were selected for further analysis. The total COP length was defined as the length of a line joining the recorded points of the COP trajectory in a 30 -second period; a higher value implied greater instability. The COP surface was measured as the surface area of an ellipse containing $90 \%$ of the recorded COP trajectory points in a 30-second period. All COP calculations were computed automatically by the platform's software.

Patients also filled in the Vertigo Syndrome Scale Short Form (VSS-SF) clinical questionnaire as proposed by Yardley et al., which has been previously used in VRT clinical trials $[1,21]$. The VSS-SF is a self-assessment where patients respond to fifteen questions addressing the frequency and severity of vertigo symptoms by ranking them from $0-4$ points. 0 points: "never", 1 point: "a few times (1-3 times a year)", 2 points: "several times (4-12 times a year)", 3 points: "quite often (on average, more than once a month)" and 4 points: "very often (on average more than once a week)". The questions group symptoms into two subsets: anxiety and balance. Severe vertigo is interpreted as 12 or more points. 
Table 1

Comparison of COP parameter change in quiet stance with eyes open before intervention and 1 month after rehabilitation

\begin{tabular}{lcccc}
\hline Training method & Parameter & $\begin{array}{c}\text { Before rehabilitation } \\
\text { (Median) }\end{array}$ & $\begin{array}{c}\text { 1 month after rehabilitation } \\
\text { (Median) }\end{array}$ & $P$-value \\
\hline Group 1 (VR) $n=25$ & COP total length [mm] & 224.1 (IQR: 192.1-310.7) & 211.1 (IQR: 171.2-282.3) & $P=0.006$ \\
& COP total surface [mm ${ }^{2}$ ] & 351.6 (IQR: 273.4-581.3) & 351.56 (IQR: 273.4-492.1) & $P=0.1$ \\
Group 2 (Control) $n=25$ & COP total length [mm] & 465.2 (IQR: 360.1-559.2) & 409.9 (IQR: 260.6-518.4) & $P=0.04$ \\
& COP total surface [mm ${ }^{2}$ ] & 546.9 (IQR: 468.8-703.1) & 532.0 (IQR: 351.6--645.3) & $P=0.24$ \\
\hline
\end{tabular}

Table 2

Comparison of COP parameter change in quiet stance with eyes closed before intervention and 1 month after rehabilitation

\begin{tabular}{lcccc}
\hline Training method & Parameter & $\begin{array}{c}\text { Before rehabilitation } \\
\text { (Median) }\end{array}$ & $\begin{array}{c}\text { 1 month after rehabilitation } \\
\text { (Median) }\end{array}$ & $P$-value \\
\hline Group 1 (VR) $n=25$ & COP total length [mm] & 387.9 (IQR: 307.1-454.0) & 300.4 (IQR: 238.3-441.4) & $P=0.001$ \\
& COP total surface [mm ${ }^{2}$ ] & 703.1 (IQR: 390.6-1024.0) & 532.1 (IQR: 340.1-742.2) & $P=0.006$ \\
Group 2 (Control) $n=25$ & COP total length [mm] & 793.4 (IQR: 633.3-966.4) & 657.3 (IQR: 556.2-793.7) & $P=0.003$ \\
& COP total surface [mm ${ }^{2}$ ] & 1054.7 (IQR: 760.9-1523.4) & 859.4 (IQR: 721.4-1054.7) & $P=0.003$ \\
\hline
\end{tabular}

Table 3

Comparison of VSS-SF scores within groups before therapy and 1 month after rehabilitation

\begin{tabular}{llcrl}
\hline Group & Subscale & $\begin{array}{c}\text { Before rehabilitation } \\
\text { (Median, IQR) }\end{array}$ & 1 month after rehabilitation & $P$-value \\
\hline Group 1 (VR) $n=25$ & Balance & $14 \mathrm{IQR}(13-18)$ & $9 \mathrm{IQR}(7-11)$ & $P=0.001$ \\
& Anxiety & $13 \mathrm{IQR}(11-15)$ & $9 \mathrm{IQR}(7-11)$ & $P=0.001$ \\
Group 2 (Control) $n=25$ & Balance & $18 \mathrm{IQR}(15-20)$ & $13 \mathrm{IQR}(11-15)$ & $P=0.001$ \\
& Anxiety & $16 \mathrm{IQR}(13-17)$ & $13 \mathrm{IQR}(11-14)$ & $P=0.001$ \\
\hline
\end{tabular}

The investigator performing the posturography assessments was not involved in implementing any aspect of the intervention, was blinded to group allocation and knew patients only by their unique study identifier. The participants were not blinded as the nature of both interventions does not allow for a double-blinded trial.

Continuous variables were summarized using median and interquartile range (IQR) for nonnormally distributed variables. The Shapiro-Wilk test was used to assess the normal distribution of continuous variables. Comparisons within groups regarding the COP parameter and VSS-SF scale change were performed using the Wilcoxon test as the variables were non-normally distributed. To analyze the effectiveness of VR rehabilitation versus the control group, the total change of COP parameters and VSS-SF score was calculated for each individual. The median values were compared. A comparison between the groups was conducted using the Mann-Whitney test for non-normally distributed variables. The level of significance used for all analyses was 2-tailed and set at $P<0.05$. Statistical analysis was performed using STATISTICA software (Version 13.1, Dell).

\section{Results}

All patients completed a full course of therapy and there were no reports of side effects.

Comparing outcomes within the groups, both the length and square surface of the COP decreased in time; however, in the quiet stance with eyes open, there was no significant change in the COP surface median (Table 1).

In a quiet stance with eyes closed, the COP parameters improved in both groups and the differences were statistically significant (Table 2 ).

Both study groups showed a lower score in the VSS-SF questionnaire regarding balance and anxiety subscales; the difference was greater in the balance subscale, which is more related to the physical perception of vertigo (Table 3 ).

The comparison of the posturographic test results between groups in eyes-open and eyes-closed conditions showed no statistically significant differences (Table 4).

The total improvement in patient-reported outcomes compared by group showed statistically significant differences in favor of VR training 
Table 4

Between the groups comparison of COP parameter differences

\begin{tabular}{lcccc}
\hline Testing conditions & Parameter & Group 1 (VR) $n=25$ (Median, IQR) & Group 2 (Control) $n=25$ (Median, IQR) & $P$-value \\
\hline Quiet stance eyes open & COP length [mm] & 36.6 (IQR: 6.0-53.8) & $41.2($ IQR: $-15.3-122.8)$ & $P=0.45$ \\
& COP surface [mm ${ }^{2}$ ] & 39.0 (IQR: -39.1-100.2) & 39.1 (IQR: $-117.2-273.4)$ & $P=0.81$ \\
Quiet stance eyes closed & COP length [mm] & $48.6(\mathrm{IQR}: 28.6-136.1)$ & $89.1(\mathrm{IQR}: 27.9-176.7)$ & $P=0.34$ \\
& COP surface [mm ${ }^{2}$ ] & $145.2(\mathrm{IQR}: 26.9-312.5)$ & 156.3 (IQR: $18.6-536.1)$ & $P=0.68$ \\
\hline
\end{tabular}

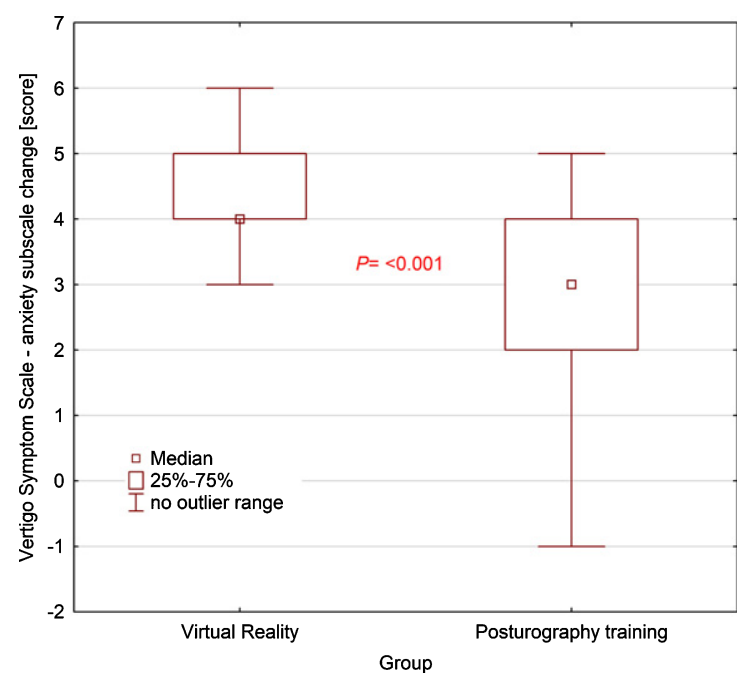

Fig. 1. Box-and-whisker plot comparing VSS-SF anxiety subscale change between groups, the $p$-value was calculated using the $\mathrm{U}$ Mann-Whitney test.

(Figs. 1-3), which was most significant in the VSS-SF subscale regarding the balance scores (Fig. 2).

\section{Discussion}

The main goal of our study was to assess whether vestibular rehabilitation utilizing hybrid VR techniques is an effective method of rehabilitation. The current results are similar to the preliminary study conducted in our Department in 2014 [11]. In the present study, the VR method was developed further by adding a force plate to the motion sensor and applying specialized software instead of commercially available VR games. Another change to the previous protocol was that all posturographic tests were performed three times upon each visit, and an average was calculated to improve the accuracy of the small study samples. Most notably, this is the first study to our knowledge to investigate a hybrid VR technique, expanding on prior VR studies.

Prior work has established VR as an effective method of vestibular rehabilitation in a popula-

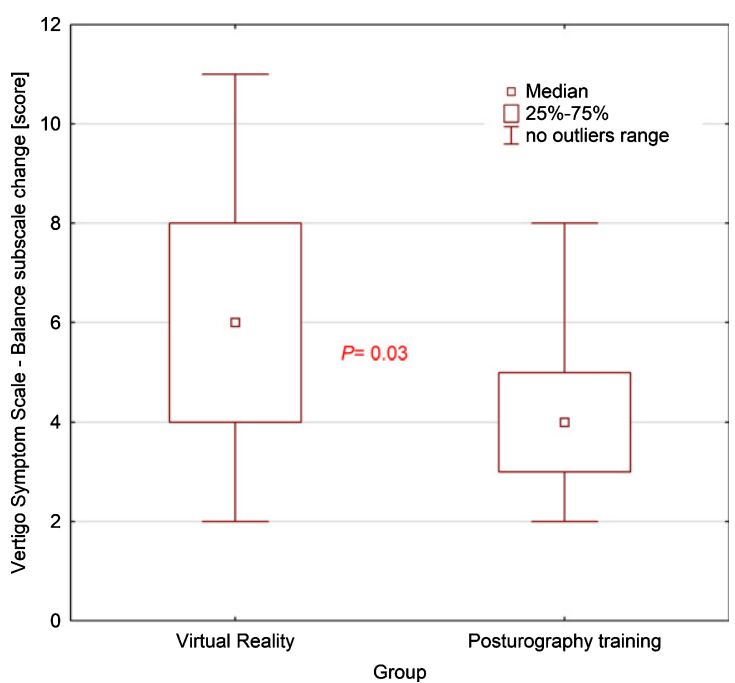

Fig. 2. Box-and-whisker plot comparing VSS-SF balance subscale change between groups, the $p$-value was calculated using the UMann-Whitney test.

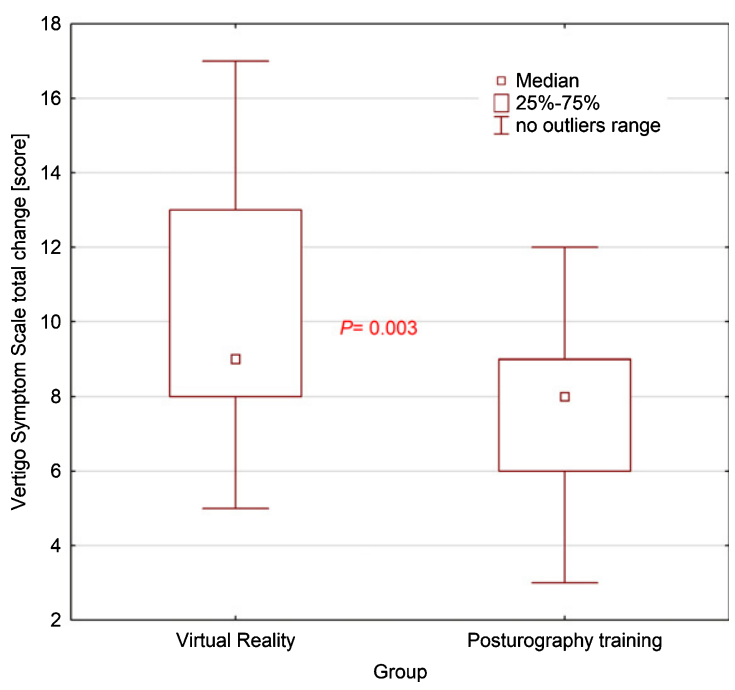

Fig. 3. Box-and-whisker plot comparing VSS-SF total change in score between groups, the $p$-value was calculated using the $\mathrm{U}$ Mann-Whitney test.

tion suffering from peripheral vestibular dysfunction; however, studies in this field remain limited. For example, Meldrum et al. [17] compared the effec- 
tiveness of conventional versus virtual reality-based (Nintendo Wii Fit Plus) vestibular rehabilitation using force-plate sensors in treating patients with unilateral peripheral vestibular loss, supporting the effectiveness of force-plate VR in these patients.

In our current study, the cumulative exposure protocol was 300 minutes per patient. Bergeron et al. [1], in their meta-analysis of VR techniques of VRT in patients suffering from vertigo of peripheral origin, emphasized that VR treatment should last at least 150 minutes of cumulated virtual environment exposure to achieve positive outcomes, and that neither the duration of a particular session nor the total number of sessions is an outcome predictive factor [1].

One study on peripheral vestibular deficiency conducted by Topuz et al. [20] concluded that significant improvement in vestibular rehabilitation in patients with poor spontaneous compensation is possible six months from the onset of symptoms. In the presented study, none of the randomized patients had achieved spontaneous recovery by two months. The two-month criterion was introduced to exclude patients with acute symptoms and to provide sufficient time for spontaneous compensation.

A systemic review by Bergeron et al. [1] expressed concern that the use of virtual reality might be limited by motion- or cybersickness because of excessive sensory stimulation. None of the patients who completed training complained of cybersickness or an exacerbation of symptoms during rehabilitation.

In objective measurements, patients show improvement in both groups, with strong statistical significance in eyes-closed conditions. Comparing the total reduction of COP length and surface between the two methods, both groups improved but neither intervention was superior. VR conditions are known to increase postural sway similar to when visual input is absent [10]. Cawthorn and Cooksey, in their original studies, recommended that VRT exercises should be performed with eyes open and closed. According to their findings, performing with eyes closed decreased the patient's reliance on visual information and probably increased the vestibular and somatosensory input to the compensation mechanisms. It is possible that, because in VR conditions the visual input is modified and different than the patient's surrounding, a similar compensatory shift occurs. Such a phenomenon might contribute to a greater improvement measured in eyes-closed conditions. Further studies with greater VR immersion using head-mounted displays are required to confirm this statement.
Posturography, while allowing quantification of postural sway or instability, is not a direct measure of vertigo sensation [12]. Self-assessment questionnaires are a part of the methodology where it is impossible to directly quantify vertigo. In our study, total improvement in both VSS-SF subscales was significantly greater for the VR group $(p<0.05)$. Thus, the overall sensation of dizziness intensity decreased more with virtual reality rehabilitation.

This study was not completely randomized, which might have resulted in potential bias and baseline differences between the groups in COP measurements. Due to the high variability of the initial COP length and surface at the baseline evaluation, a precise sample size calculation is difficult to estimate even in the presence of preliminary studies. In this study, posturography group training was related to the measurement method, which might contribute to this group achieving better results in control evaluation.

This study was underpowered; thus, certain observations might not -meet the level of statistical significance. Dynamic posturography might be a more functional method of assessing balance and could be used as an objective improvement measure for future studies.

\section{Conclusion}

Virtual reality-based vestibular rehabilitation with the application of hybrid VR units is an enjoyable and well-tolerated method of training. Hybrid VR is not superior in postural sway reduction in comparison with the established form of vestibular rehabilitation, which is static posturography with visual feedback training. However, virtual reality training seems to have a better effect on the subjective reduction of symptoms.

For future research, the authors would recommend a three-arm randomized control trial comparing the results of vestibular rehabilitation using a motion tracking VR system, a force-plate VR system and a hybrid VR unit including more outcome measurements.

\section{Acknowledgments}

The authors of this manuscript would like to express their sincerest gratitude to the staff of the Department of Rehabilitation at The Norbert Barlicki 
Memorial Teaching Hospital, Lodz, Poland in particular to Mr. Marcin Szczepanik and Mr. Jarosław Walak for their work with the patients involved in this project. The authors thank Mark Muirhead, the University of Lodz's institutional proofreader for editorial assistance. This work was performed as part of the requirements for a $\mathrm{PhD}$ degree for Oskar Rosiak. This project was supported by research funding from The National Centre for Research and Development under STRATEGMED2/266299/19/NCBR/2016.

\section{References}

[1] M. Bergeron, C.L. Lortie and M.J. Guitton, Use of Virtual Reality Tools for Vestibular Disorders Rehabilitation: A Comprehensive Analysis, Adv Med 2015 (2015), 1-9. doi: $10.1155 / 2015 / 916735$

[2] T. Cawthorne, Vestibular Injuries, J R Soc Med 39 (1946), 270-273. doi:10.1177/003591574603900522

[3] F.S. Cooksey, Rehabilitation in Vestibular Injuries, $J R$ Soc Med 39 (1946), 273-278. doi:10.1177/003591574603 900523

[4] A. Deveze, L. Bernard-Demanze, F. Xavier, J.P. Lavieille and M. Elziere, Vestibular compensation and vestibular rehabilitation. Current concepts and new trends, Neurophysiol Clin 44 (2014), 49-57. doi:10.1016/j.neucli.2013.10.138

[5] J. Diemer, A. Mühlberger, P. Pauli and P. Zwanzger, Virtual reality exposure in anxiety disorders: Impact on psychophysiological reactivity, World J Biol Psychiatry 15 (2014), 427-442. doi:10.3109/15622975.2014.892632

[6] K.R. Gottshall, P.H. Sessoms and J.L. Bartlett, Vestibular physical therapy intervention: Utilizing a computer assisted rehabilitation environment in lieu of traditional physical therapy, in: Proc Annu Int Conf IEEE Eng Med Biol Soc EMBS, (2012), pp. 6141-6144. doi:10.1109/EMBC.2012. 6347395

[7] B.I. Han, H.S. Song and J.S. Kim, Vestibular rehabilitation therapy: Review of indications, mechanisms, and key exercises, J Clin Neurol 7 (2011), 184-196. doi:10.3988/jcn. 2011.7.4.184

[8] S.J. Herdman, C.D. Hall, M.C. Schubert, V.E. Das and R.J. Tusa, Recovery of dynamic visual acuity in bilateral vestibular hypofunction, Arch Otolaryngol - Head Neck Surg $\mathbf{1 3 3}$ (2007), 383-389. doi:10.1001/archotol.133.4.383

[9] S.J. Herdman, Vestibular Rehabilitation, 2007. doi:10.1002/14651858.CD005397.pub3/abstract

[10] C.G.C. Horlings, M.G. Carpenter, U.M. Küng, F. Honegger, B. Wiederhold and J.H.J. Allum, Influence of virtual reality on postural stability during movements of quiet stance, $\mathrm{Neu}$ rosci Lett 451 (2009), 227-231. doi:10.1016/j.neulet.2008. 12.057

[11] M. Józefowicz-Korczyńska, J. Walak, M. Szczepanik, W. Lukas Grzelczyk and O. Rosiak, Ocena zastosowania wirtualnej rzeczywistości jako metody fizjoterapii w uszkodzeniu obwodowym narządu przedsionkowego, Otolaryngologia 13 (2014), 51-57.
[12] H. Kingma, G.C. Gauchard, C. De Waele, C. Van Nechel, A. Bisdorff, A. Yelnik, M. Magnusson and P.P. Perrin, Stocktaking on the development of posturography for clinical use, J Vestib Res Equilib Orientat 21 (2011), 117-125. doi:10.3233/VES-2011-0397

[13] K. Laver, S. George, S. Thomas, J. Deutsch and M. Crotty, Cochrane review: Virtual reality for stroke rehabilitation, Eur J Phys Rehabil Med 48 (2012), 523-530. doi:10.1002/14651858.CD008349.pub2

[14] L. Luna-Oliva, R.M. Ortiz-Gutiérrez, R. Cano-De La Cuerda, R.M. Piédrola, I.M. Alguacil-Diego, C. SánchezCamarero and M.D.C. Martínez Culebras, Kinect Xbox 360 as a therapeutic modality for children with cerebral palsy in a school environment: A preliminary study, NeuroRehabilitation 33 (2013), 513-521. doi:10.3233/NRE-131001

[15] D. Meldrum, A. Glennon, S. Herdman, D. Murray and R. McConn-Walsh, Virtual reality rehabilitation of balance: Assessment of the usability of the Nintendo Wii $\left({ }^{\circledR}\right)$ Fit Plus., Disabil Rehabil Assist Technol 7 (2012), 205-210. doi:10.3109/17483107.2011.616922

[16] D. Meldrum, S. Herdman, R. Moloney, D. Murray, D. Duffy, K. Malone, H. French, S. Hone, R. Conroy and R. McConnWalsh, Effectiveness of conventional versus virtual reality based vestibular rehabilitation in the treatment of dizziness, gait and balance impairment in adults with unilateral peripheral vestibular loss: A randomised controlled trial, BMC Ear, Nose Throat Disord 12 (2012), 3. doi:10.1186/1472-681512-3

[17] D. Meldrum, S. Herdman, R. Vance, D. Murray, K. Malone, D. Duffy, A. Glennon and R. McConn-Walsh, Effectiveness of Conventional Versus Virtual Reality-Based Balance Exercises in Vestibular Rehabilitation for Unilateral Peripheral Vestibular Loss: Results of a Randomized Controlled Trial, Arch Phys Med Rehabil 96 (2015), 1319-1328. doi:10.1016/j.apmr.2015.02.032.

[18] S. Moritz, M. Voigt, U. K??ther, L. Leighton, B. Kjahili, Z. Babur, D. Jungclaussen, R. Veckenstedt and K. Grzella, Can virtual reality reduce reality distortion? Impact of performance feedback on symptom change in schizophrenia patients, J Behav Ther Exp Psychiatry 45 (2014), 267-271. doi:10.1016/j.jbtep.2013.11.005

[19] H.K. Neuhauser, The epidemiology of dizziness and vertigo, 1st ed., Elsevier B.V., 2016. doi:10.1016/B978-0-44463437-5.00005-4.

[20] O. Topuz, B. Topuz, F.N. Ardiç, M. Sarhuş, G. Ogmen and F. Ardiç, Efficacy of vestibular rehabilitation on chronic unilateral vestibular dysfunction., Clin Rehabil 18 (2004), 76-83. doi:10.1191/0269215504cr704oa

[21] L. Yardley, F. Barker, I. Muller, D. Turner, S. Kirby, M. Mullee, A. Morris and P. Little, Clinical and cost effectiveness of booklet based vestibular rehabilitation for chronic dizziness in primary care: Single blind, parallel group, pragmatic, randomised controlled trial, BMJ 344 (2012). doi:10.1136/bmj.e2237 